\title{
Post Traumatic Internal Derangement of Knee Evaluation at 3.0 Tesla MRI
}

\author{
Indrajeet Kumar ${ }^{1}$, Neeraj Prajapati ${ }^{2}$, Asif Muzaffer Reshi ${ }^{3}$ \\ ${ }^{1}$ Junior Resident-III, Department of Radiodiagnosis, 2 Professor, Department of Radiodiagnosis, ${ }^{3}$ Senior Resident, Department \\ of Radiodiagnosis, Shri Ram Murti Smarak Institute of Medical Sciences, Bareilly, India
}

Corresponding author: Dr. Neeraj Prajapati, SRMSIMS, Bhojipura, Bareilly, India

DOI: http://dx.doi.org/10.21276/ijcmsr.2020.5.2.5

How to cite this article: Indrajeet Kumar, Neeraj Prajapati, Asif Muzaffer Reshi. Post traumatic internal derangement of knee evaluation at 3.0 tesla MRI . International Journal of Contemporary Medicine Surgery and Radiology. 2020;5(2):B17-B20.

\section{A B S T R A C T}

Introduction: Radiological evaluation of internal derangement of the knee joint using 3.0 Tesla Magnetic Resonance Imaging in cases of knee trauma.To study the pattern of internal derangement of knee on 3.0 Tesla Magnetic Resonance Imagining in cases of knee trauma. Setting \& design: A descriptive statistical analysis and correlation evaluation study consisting of 60 patients with Traumatic knee joint was undertaken to study the spectrum of MRI findings in all consecutive cases of knee trauma referred from orthopedic OPD and Comparison of findings of magnetic resonance imaging .

Materials and methods: This study was conducted on Siemens Skyra 3.0 Tesla 48 channel MRI and X-Ray Siemens Polydoros LX -800 MA machines in the department of Radio diagnosis at SRMSIMS Bareilly in patients presented with knee trauma. All the sequences were performed in all the patients and data collected.

Results: The most common injury was ACL injury ( 91\%). Among the Meniscal injuries lateral meniscus were less common than Medial Meniscal tears and grade III tears were common in both. Medial collateral ligament tears outnumbered Lateral ligament tears and grade III tears were more common in Medial collateral ligament.

Conclusion: Magnetic resonance imaging has emerged as the frontline investigation for evaluation of internal derangements of the knee joint. It is a noninvasive procedure and it does not involve ionizing radiation. It also has Multiplanner capability with great soft tissue demonstration. Arthroscopy gives great anticipation of the interior of the joint, but is invasive and can only evaluate the surface abnormalities.

Keywords: Anterior Cruciate Ligament, Magnetic Resonance Imaging, Medial Collateral ligament, Medial Meniscus, Lateral Meniscus, Posterior Cruciate Ligament

\section{INTRODUCTION}

Internal derangement of the knee is an umbrella term which is used to cover a group of disorders comprises destruction of the normal functioning of the ligaments and/or cartilages. Magnetic Resonance Imaging provides an very good soft tissue contrast and is highly effective in evaluation of the soft tissue and bony structures in multiple imaging planes which provide considerable advantage in evaluation of IDK. ${ }^{1}$ The knee being the largest joint in the human body withstands complex forces which are exerted during various activities, rendering it prone to a number of acute and chronic injuries. Trauma to the knee is a substantial cause of disease in the young, active individuals particularly amongst military personnel's and athletes. MRI and arthroscopy are the most extensively used diagnostic modalities to evaluate the joint injury. Arthroscopy, nonetheless accurate, is invasive and may cause complications. ${ }^{2,3}$ Meniscal and ligament injuries this joint can be evaluated by means of magnetic resonance imaging (MRI) examinations, which provide images showing abnormalities of the morphology that are characterized. Magnetic Resonance Imaging is a considerable advance and may well preserve patients from unnecessary operations. IDK is evaluated with radiography, MRI, arthrography and arthroscopy. Arthroscopy is generally considered as the gold standard for the evaluation of knee injury. However, the accuracy of arthroscopy varies from $69 \%$ to $98 \%$ depending of the experience of examiner. Also, intrasubstance tears cannot be evaluated in arthroscopy.

\section{MATERIAL \& METHODS}

This study was conducted on Siemens Magnetic Resonance 3.0 Tesla 48 channel MRI (Siemens Skyra) and X-Ray Siemens Polydoros LX -800 MA machines in the department of Radio diagnosis at SRMSIMS Bareilly. All patients of different age groups referred for MRI with history of trauma to the knee joint was included in this study were included in the study. Post - operative patients, Patients with ferromagnetic implants, pacemakers and aneurysm clips were excluded from the study. A complete history of the patient was taken. 
The radiographs were performed in both AP and lateral views. Then a multiplanar MRI was performed. The sequences performed were fat sat proton density $(\mathrm{PD})$ in axial, coronal and saggital planes, T1 in saggital and coronal planes, T2 in axial plane with a slice thickness of $3 \mathrm{~mm}$. A volumetric 3D gradient sequence for evaluation of bony pathologies (specially the avulsion fractures) was also performed in the end of the study. The standard TR, TE and other parameters as that are applicable on a $3 \mathrm{~T} \mathrm{MRI} \mathrm{were} \mathrm{applied.}$ The radiographs and MRI were read by MRI consultant having more than five years experience in musculoskeletal MRI.

\section{RESULTS}

A total of 60 patients were included in the study. Out of 60 patients 7 were in age group of $\leq 20$ years, 14 each were in both age group of 21-30 years and 31-40 years, 13 were in age group of 41-50 years and 12 were in age group of $>50$ years. Mean age of patients were $37.4 \pm 14.6$ years. Complaint of pain and swelling were found in all 60patients.Knee joint effusion was present in majority of patients. ACL Tears were present in 55 patients out of which 30 had partial and $25 \mathrm{had}$ complete ACL Tear.PCL Tears were present in 21 patients out of which 13 had partial and 8 had complete PCL Tear.Out of 60 patients MCL Tears were present in only 10patients 4 had grade 1, 2 had grade 2 and 4 had grade 3 tears.LCL Tears were present in only 15 patients, 9 had grade 1 , 4 had grade 2 and 2 had grade 3 tears. Out of 60 patients MM Tear were present in 25 patients, 09 patients had Complex tear 1 had Radial tear, 2 had Horizontal tear, 4 had Bucket Handle tear 1 and Oblique tear in 1 patient respectively (Table 1).

\begin{tabular}{|l|c|c|}
\hline Spectrum of MRI findings & Number & Percentage (\%) \\
\hline Joint Effusion & 48 & 80.0 \\
\hline ACLTear & 55 & 91.7 \\
\hline PCLTear & 21 & 35.0 \\
\hline MCLTear & 10 & 16.7 \\
\hline LCLTear & 15 & 25.0 \\
\hline MM Tear & 25 & 41.66 \\
\hline LM Tear & 11 & 18.3 \\
\hline Osseous/Osteochondral lesions & 28 & 46.7 \\
\hline \multicolumn{2}{|c|}{ Table-1: Spectrum of MRI findings } \\
\hline \multicolumn{2}{|c|}{} \\
\hline
\end{tabular}
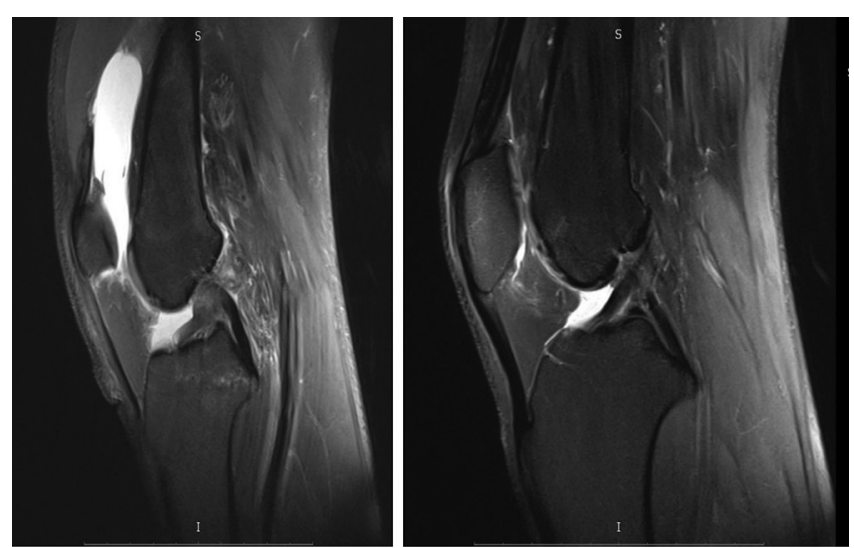

Figure-1: Complete ACL tear; Figure-2: Partial ACL tear near tibial insertion

\section{DISCUSSION}

The role of MRI has greatly increased and now it has become the first line investigation for most of the knee lesions. It is also being used for pre and post operative evaluation.

Anterior cruciate ligament (ACL): attaches the tibia \& the femur bone. It is located deep inside the knee and is in front of the posterior cruciate ligament. It mainly functions to limit the forward movement of the tibia relative to the femur. It also restricts some rotation and sideways motion of the knee.

Injuries of Anterior cruciate ligament (ACL): The ACL can be torn with sudden pivoting motions of the knee. ${ }^{4}$ It can have both complete and partial tears.MRI signs of complete tears - The direct signs include ${ }^{5,6,7}$ i) Discontinuity of ACL fibers.(Fig- 1). ii) Abnormal contour of anterior cruciate ligament. The indirect signs include ${ }^{5,8}$ i) Buckling of PCL. ii) kissing bone contusion. iii) Overhanging of posterior horn of the lateral meniscus. iv) Segond fracture of the lateral tibia v) Deep lateral femoral sulcus exceeding $2 \mathrm{~mm}$ in depth. vi) Chip fracture of the posterior tibia vii) Anterior translation of tibia. Subacute phase is the best time for evaluation of tear. Proximal end may be displaced posterior to the PCL giving the appearance of a loose body.

The partial ACL tears presented as the thickening and hyperintensity of the ACL fibres with maintained continuity.(Fig- 2).

Chronic anterior cruciate ligament tears are tough to evaluate due to accompanying atrophy. The distal end of the Anterior Cruciate Ligament may be seen attached to the posterior cruciate ligament without anticipation of the proximal end and is commonly reported as normal.

Posterior cruciate ligament (PCL) - like the Anterior Cruciate Ligament, it also attaches the tibia and the femur. It lies behind the ACL. It mainlyrestricts backward motion of the tibia in relation to the femur. Like the Anterior Cruciate Ligament, it also limits some rotation and some of the sideways motion of the knee. The PCL can be torn with a forceful landing on the shin. ${ }^{3}$

Injuries of Posterior cruciate ligament (PCL): MRI appearances comprise thickening of the middle portion of the ligament with hyperintensities on both T1- and T2weighted images. Continuity of the ligament is maintained in the PCL with acute trauma, unlike acute anterior cruciate ligament tears. ${ }^{7,9}$ Chronic posterior cruciate ligament tears are difficult to identify because the ligament will form a fibrous scar in the place of normal ligament which is of same signal intensity to the rest of the ligament. Secondary signs include ligament laxity or persistent hyperintensity. ${ }^{7,10}$ (figure-3,4).

\section{Medial collateral ligament}

The principal component of the intermediate layer is the superficial portion of the MCL. The superficial portion of the medial collateral ligament is found along the middle third of the knee and is composed of vertically oriented fibers. It is also referred to as the vertical component of the MCL. Posteriorly with regard to the vertical component of the $\mathrm{MCL}$, the posterior oblique portion of the MCL is found. 
This posterior oblique portion of the MCL is fused with layer 3 and closely attached to the posteromedial meniscus. ${ }^{11}$

Medial collateral ligament (MCL) tears Grading of MCL tears; Grade I: Intact Medial Collateral Ligament fibers with hyperintensity, superficial to the medial collateral ligament due to edema. Grade II: Partial tear of Medial collateral ligament Grade III: Complete tear of the MCL fibers with associated edema. Complete Medial collateral ligament tear (figure-4).

\section{Lateral collateral ligament}

The lateral side of the knee is stabilized by acomplex arrangement of ligaments, tendons, \& muscles. These structures provide the knee with anterolateral and posterolateral stabilization. Lateral collateral ligament (LCL) LCL injuries are mainly associated with other injuries. ${ }^{12}$ The common injuriesassociatedare the injuries of the capsule, the biceps femoris, and the popliteus, less commonly also cruciate ligaments or the lateral tibial rim (Segond fracture) and usually as a part of posterolateral corner injury. ${ }^{13,14}$ (figure-5).

\section{Menisci}

The menisci function is to absorb shock, transmit axial load, assist in joint lubrication, and facilitate nutrient distribution. The Medial Menisci and Lateral Menisci are wedge-shaped, semilunar, fibrocartilaginous structures. Each meniscus has a superior concave surface that confors to the femoral condyle and a flat base that fixed to the tibia via the central root ligaments. Each of the menisci can be subdivided into an anterior horn, body, posterior horn, and the roots. The

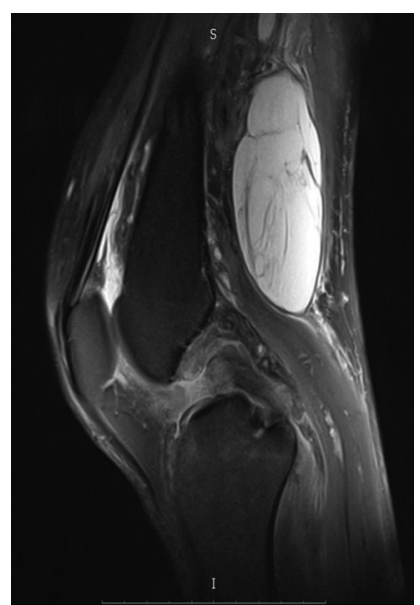

Figure-3: Complete PCL tear
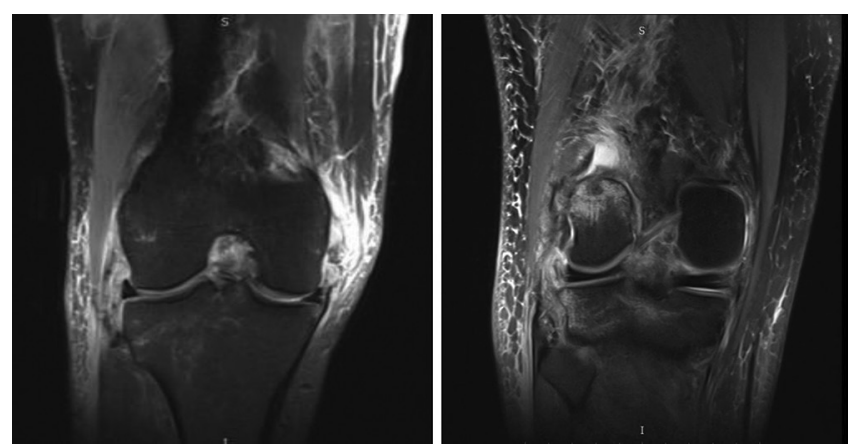

Figure-4: Complete MCL tear; Figure-5: LCL complete tear anterior and posterior roots generally attach to the central tibial plateau, acting as the anchors and maintaining the normal meniscal position \& biomechanical function. ${ }^{15}$ Compression and rotation with abnormal shearing forces of knee results in meniscal damage. Clinically patient may present with joint pain, giving way, clicking, locking of knee in fixed flexion and swelling.

\section{Medial Meniscus}

The medial meniscus is a crescent (moon) shaped structure that is present on the inside of the knee. It is made of fibro-cartilage. It acts as a shock absorber in the knee and providesstability to the knee joint. It is attached to the tibia and also to the joint capsule of the knee. ${ }^{4}$

\section{Lateral Meniscus}

The lateral meniscus sits on the lateral tibial plateau. It is a crescent shaped structure that is made up of fibrocartilage. It also acts as a shock absorber in the knees and provides extra stability to the knee joint. It is joint to the capsule of the knee joint also. It is somewhat more mobile than the medial meniscus.

Meniscal Tears: Diagnostic Criteria: Two criteria for diagnosing a meniscal tear are commonly used ${ }^{16}$ :i) An hyperintense intrasubstance area extending to the articular surface, or ii) Abnormal meniscal morphology.

Classification meniscal of tears: Horizontal, Radial, Vertical longitudinal, Horizontal flap, Vertical flap, Complex and Bucket handle tears. (Fig 6-9)

Discoid Meniscus, Meniscal Ossicles and Meniscal flounce are normal variants. ${ }^{8,18}$

Grading of meniscal injury Grade 1: Intrameniscal hyperintensity not extending to the articular surface. Grade 2: Linear signal intensity that does not intersect the inferior or superior articular surface but is in contact with the capsular margin at the posterior aspect of the meniscus. Grade 3: Hyperintensity extending to the superior and/ or inferior articular surface. Grade 1 and grade 2 lesions indicate mucinous or mucoid intrasubstance degenerative change and are usually encountered after 3 or 4 decade. Due to prominent vascularity in children and young adults misinterpretation as grade 1 or 2 tear is usual. ${ }^{8,17}$

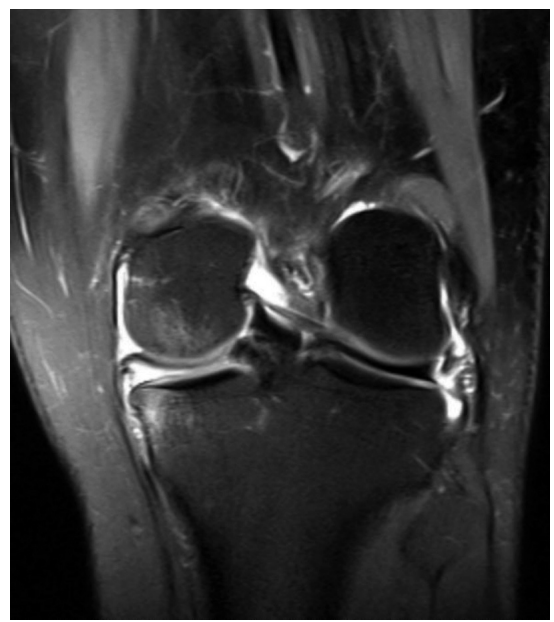

Figure-6: Bucket handle of post. horn of medial meniscus 

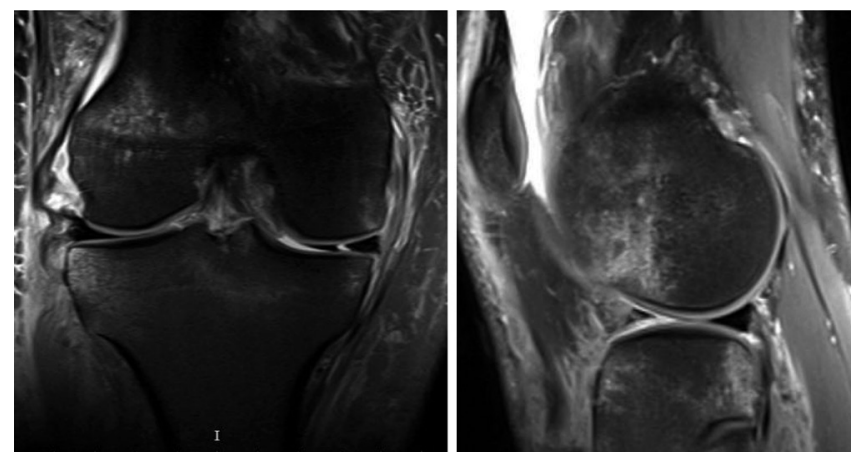

Figure-7: Longitudinal tear of medial meniscus; Figure-8: Complex meniscal tear of anterior horn

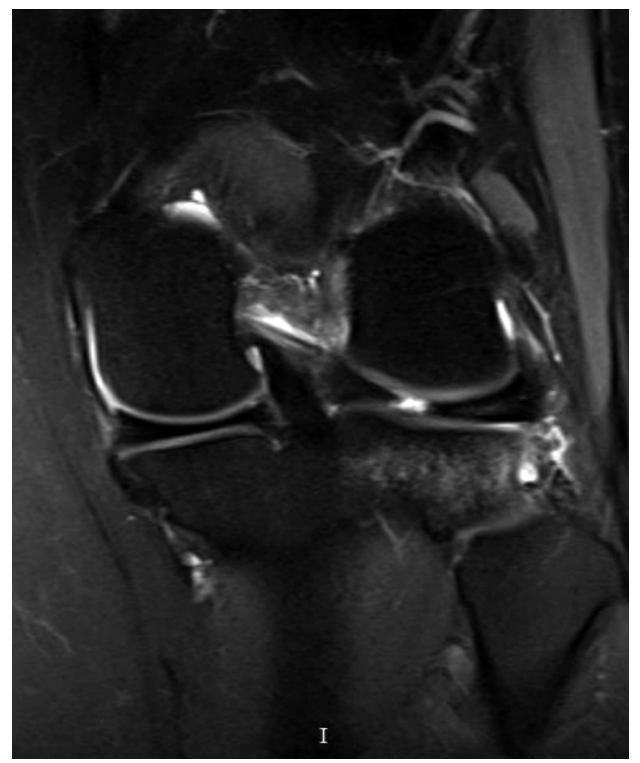

Figure-9: Radial tear of post. horn of lateral meniscus

\section{CONCLUSION}

The present study attempts to determine the role of magnetic resonance imaging in the evaluation of internal derangements of the knee joint.

Magnetic resonance imaging has emerged as the gold standard investigation for evaluation of internal derangements of the knee joint.

It is a noninvasive technique, does not involve ionizing radiation and has Multiplanner capability with a great soft tissue demonstration. Arthroscopy gives great visualization of the interior of the joint, but is invasive and can also evaluate the surface abnormalities.

\section{REFERENCES}

1. Arumugam V, Ganesan GR, Natarajan P. MRI Evaluation of Acute Internal Derangement of Knee. Open Journal of Radiology. 2015;5(02):66.

2. Werman, Howard A.; Robert Paasche,; M. Ott, Knee trauma: assessment, diagnostic evaluation, and management. Pediatric Emergency Medicine Reports. AHC Media L.L.C. 2004.

3. Rangappa Hanumappa Chavadaki, Praveen Kumar Reddy P, Reneesh UruniyanParamban, Swetha Gurram. MRI of the internally deranged knee joint. A Prospective study. Journal of Evolution of Medical and
Dental Sciences 2013; 2(42): 8186-8206.

4. Chung A: Knee Anatomy, function and common problems. Health pages. Org. 2019, march 30.

5. Crema MD, Hunter DJ, Roemer FW, et al. The relationship between prevalent medial meniscal intrasubstance signal changes and incident medial meniscal tears in women over a 1 year period assessed with 3.0

6. Marc A. Camacho. The Double Posterior Cruciate Ligament Sign. Radiology 2004; 233(6):503- 504.

7. Sonin AH, Fitzgerald SW, Friedman H: MR imaging of Posterior cruciate ligament; Normal, Abnormal and associated injury pattern. Radiographics 1995; 15(2): 551-561.

8. Ryu KN, Kim IS, Kim EJ, et al. MR imaging of tears of discoid lateral menisci. AJR 1998; 171(2):963-967.

9. Grover JS, Bassett LW, Gross ML. Posterior cruciate ligament: MR imaging. Radiology 1990;174(5):527530.

10. Akisue T, Kurosaka M, Yoshiya S. Evaluation of healing of the injured posterior Cruciate Ligament: analysis of Instability and Magnetic Resonance Imaging. Arthroscopy 2001;17(3): 264-269.

11. Maeseneer MD, Roy FV, Lenchik L. Three layers of the medial capsular and supporting structures of the knee: MR imaging-Anatomic correlation. Radiographics 2000; 20(1):S83-S89.

12. Miller TT, Gladden P, Staron RB, Henry JH, Feldman F: Posterolateral Stabilizers of the knee: anatomy and injuries assessed with MR imaging. AJR Am J Roentgenol 1997;169(6):1641-1647.

13. Edwin H G Oei, Abida Z Ginai, M G MyriamHunink. MRI for Traumatic Knee Injury: A Review. Semin Ultrasound CT MRI 2007;28(4):141-157.

14. Mink JH: The Cruciate and Collateral ligaments, in Mink JH, Reicher MA, Crues JV III, (eds): MRI of the Knee (2nd ed). New York, Raven, 1993, pp 141- 188.

15. Fie CN, Arthur A, Ben K, Humberto G. MR Imaging - based diagnosis and classification of Meniscal Tears. Radiographics 2014; 34(2):981-999.

16. Edwin H G Oei, Jeroen J Nikken, Antonia C M Verstijnen, Abida Z Ginai, M G Myriam Hunink.MR Imaging of the Menisci and Cruciate Ligaments: A Systematic Review. Radiology2003;226(6):837- 848.

17. Samoto N, Kozuma M, Tokuhisa T, et al. Diagnosis of Discoid Lateral Meniscus of the Knee on MR imaging. Magn Reson Imaging 2002; 20(1):59-64.

18. Michael G. Fox, MR Imaging of the Meniscus: Review, Current Trends, and Clinical Implications; radiolclin N Am 2007; 45(1):1033-1053.

Source of Support: Nil; Conflict of Interest: None

Submitted: 14-02-2020; Accepted: 13-04-2020; Published online: 10-05-2020 